\title{
Identifying potential biomarkers of nonalcoholic fatty liver disease via genome-wide analysis of copy number variation
}

Yang fan $\mathrm{Li}^{1+}$, Jing Zheng ${ }^{1 \dagger}$, He wei Peng ${ }^{1}$, Xiao lin Cai ${ }^{1}$, Xin ting Pan ${ }^{1}$, Hui quan Li', Qi zhu Hong ${ }^{1}$, Zhi jian Hu${ }^{1}$, Yun li Wu ${ }^{2^{*}}$ and Xian-E. Peng ${ }^{1,2^{*}}$

\begin{abstract}
Background: The prevalence of Non-alcoholic fatty liver disease (NAFLD) is increasing and emerging as a global health burden. In addition to environmental factors, numerous studies have shown that genetic factors play an important role in the development of NAFLD. Copy number variation (CNV) as a genetic variation plays an important role in the evaluation of disease susceptibility and genetic differences. The aim of the present study was to assess the contribution of CNV to the evaluation of NAFLD in a Chinese population.
\end{abstract}

Methods: Genome-wide analysis of CNV was performed using high-density comparative genomic hybridisation microarrays (ACGH). To validate the CNV regions, TaqMan real-time quantitative PCR (qPCR) was utilized.

Results: A total of 441 CNVs were identified, including 381 autosomal CNVs and 60 sex chromosome CNVs. By merging overlapping CNVs, a genomic CNV map of NAFLD patients was constructed. A total of 338 autosomal CNVRs were identified, including 275 CNVRs with consistent trends (197 losses and 78 gains) and 63 CNVRs with inconsistent trends. The length of the 338 CNVRs ranged from $5.7 \mathrm{~kb}$ to $2.23 \mathrm{Mb}$, with an average size of $117.44 \mathrm{~kb}$. These CNVRs spanned $39.70 \mathrm{Mb}$ of the genome and accounted for $~ 1.32 \%$ of the genome sequence. Through Gene Ontology and genetic pathway analysis, we found evidence that CNVs involving nine genes may be associated with the pathogenesis of NAFLD progression. One of the genes (NLRP4 gene) was selected and verified by quantitative PCR (qPCR) method with large sample size. We found the copy number deletion of NLRP4 was related to the risk of NAFLD.

Conclusions: This study indicate the copy number variation is associated with NAFLD. The copy number deletion of NLRP4 was related to the risk of NAFLD. These results could prove valuable for predicting patients at risk of developing NAFLD.

Keywords: Nonalcoholic fatty liver disease, Copy number variation, Case control study

*Correspondence: wuyinli422@163.com; fmuxe@163.com

†Yang fan Li and Jing Zheng contributed equally to this work

${ }^{1}$ Department of Epidemiology and Health Statistics, Fujian Provincial Key Laboratory of Environment Factors and Cancer, School of Public Health,

Fujian Medical University, Fuzhou 350108, China

2 Key Laboratory of Ministry of Education for Gastrointestinal Cancer,

Fujian Medical University, Fuzhou 350108, China

\section{Introduction}

Non-alcoholic fatty liver disease (NAFLD), defined as surplus fat accumulated in the liver, covers a spectrum from simple steatosis to non-alcoholic steatohepatitis (NASH), fatty liver fibrosis (HF), liver cirrhosis (LC) and hepatocellular carcinoma (HCC) [1]. NAFLD is also closely associated with higher prevalence of obesity, type 
2 diabetes and cardiovascular disease [2-4]. The prevalence of NAFLD is increasing and emerging as a global health burden, therefore it is crucial to explore pathogenesis and effective prevention management.

In addition to environmental factors, numerous studies have shown that genetic factors play an important role in the development of NAFLD. For example, genomewide association studies (GWAS) revealed that singlenucleotide polymorphisms of genes are closely related to the risk of NAFLD [5-7]. Copy number variation (CNV) covers submicroscopic variation in the human genome ranging from 1 to $3 \mathrm{Mb}$ in size, and includes insertions, inversions, deletions, duplications and translocations. $\mathrm{CNV}$ includes gene coding regions and regulatory elements that can influence gene expression, phenotypic variation and adaptation via disruption of genes, and altering gene dosage [8]. Studies have shown that CNV plays an important role in the evaluation of disease susceptibility and genetic differences in Alzheimer's disease [9], Parkinson's disease [10], schizophrenia [11], liver cancer [12] and lung cancer [13]. However, studies on the association between CNV and NAFLD are limited, there exists genetic heterogeneity among study populations, and the sample size is limited.

Herein, a case-control study was designed and conducted in which array-based comparative genomic hybridisation (ACGH) was performed to identify potential CNV associated with NAFLD. Gene annotation analysis software was utilised to ascertain biological processes associated with genes related to CNV. The findings may provide epidemiological evidence for the diagnosis and prevention of NAFLD.

\section{Materials and methods}

\section{Subjects}

The study was approved by local ethics committees of Fujian Medical University (ethics number 2014096). Subjects were recruited from the Health Examination Centre of Nanping First Affiliated Hospital of Fujian Medical University from October 2015 to September 2017. Once cases and controls were linked to NAFLD, a letter of invitation and information about the study was sent to each potential case and control to obtain consent. In order to standardize the experimental process, improve the accuracy of the results, and enhance the comparability of the conclusions, all methods are implemented in accordance with relevant guidelines and regulations.

All subjects underwent abdominal ultrasound, and NAFLD was diagnosed by the presence of at least two of the following three abnormal findings following abdominal ultrasonography [14]: (1) increased echogenicity of the liver near-field region with deep attenuation of the ultrasound signal; (2) hyperechogenity of liver tissue ('bright liver'), accompanied by hypoechogenicity of the kidney cortex; (3) vascular blurring.

\section{Clinical and laboratory evaluation}

Demographic and anthropometric criteria were assessed for NAFLD patients and normal controls, including sex, age, body mass index (BMI; weight $[\mathrm{kg}] /$ height $\left.\left[\mathrm{m}^{2}\right]\right)$, and waist-to-hip ratio $[$ WHR, waist circumference $[\mathrm{cm}] /$ hip circumference $[\mathrm{cm}]$ ). Various biochemical tests were performed, including fasting blood glucose (FBG), total cholesterol (TC), triglycerides (TG), high-density lipoprotein cholesterol (HDL-C), low-density lipoprotein cholesterol (LDL-C), alanine transaminase (ALT) and aspartate transaminase (AST).

\section{ACGH and CNV calling}

Based on liver fatty accumulation diagnosed by abdominal ultrasound, NAFLD was divided into three grades, and four cases were selected from each grade [15]. The matching principle was formulated according to the gender, age ( \pm 5 years). The controls was selected from healthy people diagnosed with no NAFLD by abdominal ultrasound during the same period. And those who met the case exclusion criteria were not included. Exclusion criteria were as follows: (1) alcohol consumption $>140 \mathrm{~g} /$ week for men and $>70 \mathrm{~g} /$ week for women; (2) the presence of hepatitis B surface antigens or hepatitis $C$ antibodies; (3) use of hepatotoxic drugs (such as tamoxifen, amiodarone, valproate and methotrexate) that can induce hepatic fat accumulation [16]; (4) hepatic disease, which can induce hepatic fat accumulation; (5) hepatic diseases such as Wilson's disease, autoimmune hepatitis and hemochromatosis. ACGH was performed on 12 NAFLD patients and 12 healthy controls.

We mainly focus on genetic susceptibility between CNV and NAFLD in the present study, DNA mutations in peripheral leukocytes reflect germline mutation, suggesting the association between mutations and genetic susceptibility, therefore peripheral leukocyte blood was utilised. Genomic DNA was extracted from peripheral blood samples obtained from each subject using a nucleic acid extraction kit (Qiagen, Hilden, Germany). DNA quality was determined by a Denovix DS-11 spectrophotometer (Denovix, Waltham, MA, USA). DNA purity was verified by A260/A280 ratio of 1.80-2.0. ACGH was performed according to the protocol established by the manufacturer (Oxford Gene Technology, Begbroke, UK). It was carried out using SurePrint G3 Human Genome $4 \times 180 \mathrm{~K}$ microarrays (Agilent Technologies, Santa Clare, CA, USA) for genome-wide identification of putative disease-associated CNVs. The microarrays contained $\sim 180,000$ probes that enabled the profiling of molecular genomic imbalance with a mean resolution of 
$13 \mathrm{~kb}$. Probes in the array covered both coding and noncoding regions of the human genome. A total of $1 \mu \mathrm{g}$ genomic DNA from patients and controls was labelled with $\mathrm{Cy} 3$ and $\mathrm{Cy} 5$ dyes, respectively and probes were purified and mixed thoroughly using an Agilent SureTag Complete DNA Labeling Kit (Agilent Technologies). This was followed by denaturation and pre-annealing with $50 \mu \mathrm{g}$ human Cot-1 DNA. Hybridisation of the mixture was performed on an array slide with constant rotation at $65{ }^{\circ} \mathrm{C}$ for $40 \mathrm{~h}$. The slide was then washed with Agilent wash buffers 1 and 2, and scanned immediately using an Agilent G2565CA Microarray Scanner (Agilent Technologies). Data were extracted from scanned images using Feature Extraction Software version 10.10 (Agilent Technologies). Raw data were uploaded into Agilent Cytogenomics software (Agilent Technologies). Genomic aberrations were identified by applying $\log 2$ intensity ratios of samples to references (Cy3/Cy5, log2 ratios above 0 for duplicates, and below 0 for deletions). $\mathrm{CNV}$ was assigned for segments with at least three consecutive probes. Chromosomal aberrations were reported in accordance with the human genome sequence assembly Build 37, hg 19 (http://www.ncbi.nlm.nih.gov).

\section{Functional enrichment analysis of CNV}

After merging overlapping CNVs appearing in two or more samples, a genomic CNV map of NAFLD patients was constructed using $R$ software. Genes associated with CNV were retrieved from the Homo sapiens (GRCh37) assembly provided by University of California Santa Cruz (UCSC). To analyse genes with CNV and investigate the functional impact of $\mathrm{CNV}$ on various biological processes, KOBAS gene annotation analysis software which can be accessed at http://kobas.cbi.pku.edu.cn was employed. This program annotates an input set of genes with putative pathways and disease relationships based on mapping to genes with known annotations. It allows for both ID mapping and cross-species sequence similarity mapping. It then performs statistical tests to identify statistically significantly enriched pathways and diseases. KOBAS 2.0 incorporates knowledge across 1327 species from five pathway databases (KEGG PATHWAY, PID, BioCyc, Reactome and Panther) and five human disease databases (OMIM, KEGG DISEASE, FunDO, GAD and NHGRI GWAS Catalog) [17]. The final list of genes associated with NAFLD was determined using the Pubmed database.

\section{Quantitative PCR validation of CNV calls}

To validate the CNV regions, TaqMan real-time quantitative PCR (qPCR) was performed using a Step One Plus instrument (Applied Biosystems) on 557 samples (297 cases and 260 controls) from one selected region (19q13;
Assay Hs02992963_cn) which contained the NLRP4 gene. Each reaction $(20 \mu \mathrm{l})$ contained $10 \mu \mathrm{l}$ master mix, $1 \mu \mathrm{l}$ TaqMan Copy Number Assay, $1 \mathrm{ml}$ TaqMan Copy Number Reference Assay, $4 \mu \mathrm{l}$ nuclease-free water, and 4 $\mu \mathrm{l} 5 \mathrm{ng} / \mu \mathrm{l}$ genomic DNA. All reactions were performed in quadruplicate. Thermal cycling conditions consisted of an initial denaturation step at $95^{\circ} \mathrm{C}$ for $10 \mathrm{~min}$, followed by 40 cycles at $95{ }^{\circ} \mathrm{C}$ for $15 \mathrm{~s}$ and $60{ }^{\circ} \mathrm{C}$ for $1 \mathrm{~min}$. Furthermore, we analysed CNV in the NLRP4 gene and its association with the risk of NAFLD.

\section{Statistical analysis}

Chi-square tests were employed to assess categorical variables, and Mann-Whitney U tests were used for continuous variables. An unconditional logistical regression model was employed to analyse the association between target gene CNV and NAFLD risk. All statistical analyses were performed by SPSS 23.0 software (SPSS, Inc, Chicago, IL, USA). A two-tailed $p$-value $<0.05$ was considered statistical significant.

\section{Results}

Clinical characteristics of the study population

The clinical characteristics of the study population are outlined in Table 1. Compared with those of the control group, levels of BMI, WHR, ALT, TG, TC, FBG and LDL-C were significantly higher in the NAFLD group $(p<0.01-0.05)$ (Table 1$)$.

\section{Detailed features of CNVRs in the genome}

A rigorous quality control check was performed during sample processing, and all DNA samples were suitable for study. In total, $441 \mathrm{CNVs}$ were detected, including 381

Table 1 Clinical characteristics of NAFLD patients and normal controls

\begin{tabular}{llll}
\hline Characteristics & NAFLD $(n=12)$ & Controls $(n=12)$ & $p$-value \\
\hline Gender (F/M) & $9 / 3$ & $9 / 3$ & 1 \\
Age (years) & $41.33 \pm 11.36$ & $41.00 \pm 11.33$ & 0.84 \\
WHR & $0.92 \pm 0.05$ & $0.83 \pm 0.04$ & $<0.001$ \\
BMI $\left(\mathrm{kg} / \mathrm{m}^{2}\right)$ & $26.83 \pm 2.78$ & $21.44 \pm 1.70$ & $<0.001$ \\
ALT & $45 \pm 48.20$ & $12.25 \pm 4.50$ & 0.001 \\
AST & $28.5 \pm 18.48$ & $19.5 \pm 2.84$ & 0.31 \\
TC & $5.81 \pm 0.70$ & $4.67 \pm 0.28$ & $<0.001$ \\
TG & $2.07 \pm 1.18$ & $0.82 \pm 0.24$ & $<0.001$ \\
FBG & $5.57 \pm 0.67$ & $4.98 \pm 0.33$ & 0.009 \\
LDL-C & $3.73 \pm 0.63$ & $2.97 \pm 0.18$ & 0.001 \\
HDL-C & $1.29 \pm 0.18$ & $1.33 \pm 0.21$ & 0.435 \\
\hline
\end{tabular}

$B M I$ body mass index, WHR waist-to-hip ratio, AST serum aspartate aminotransferase, ALT alanine aminotransferase, GGT gammaglutamyltranspeptidase, $F B G$ serum fasting glucose, $T C$ total cholesterol, $T G$ triglycerides, LDL-C low-density lipoprotein, $H D L-C$ high-density lipoprotein 
autosomal CNVs and 60 sex chromosome CNVs (Fig. 1). Owing to the evolutionary bias due to small imbalances of sex chromosomes, we opted to exclude sex chromosome CNVs from further analysis. The 381 autosomal CNVs spanned between $5.7 \mathrm{~kb}$ and $2.35 \mathrm{Mb}$ in size, with an average size of $181.8 \mathrm{~kb}$ (Table 2 and Additional file 1:
Table SI). All samples displayed both copy number gains and losses, but copy number gains were more commonly observed than losses (estimated ratio of 1.7:1).

Any CNVs overlapping between two or more samples were defined as shared CNVs, and these were integrated, longer fragments were split, and a genomic CNV map

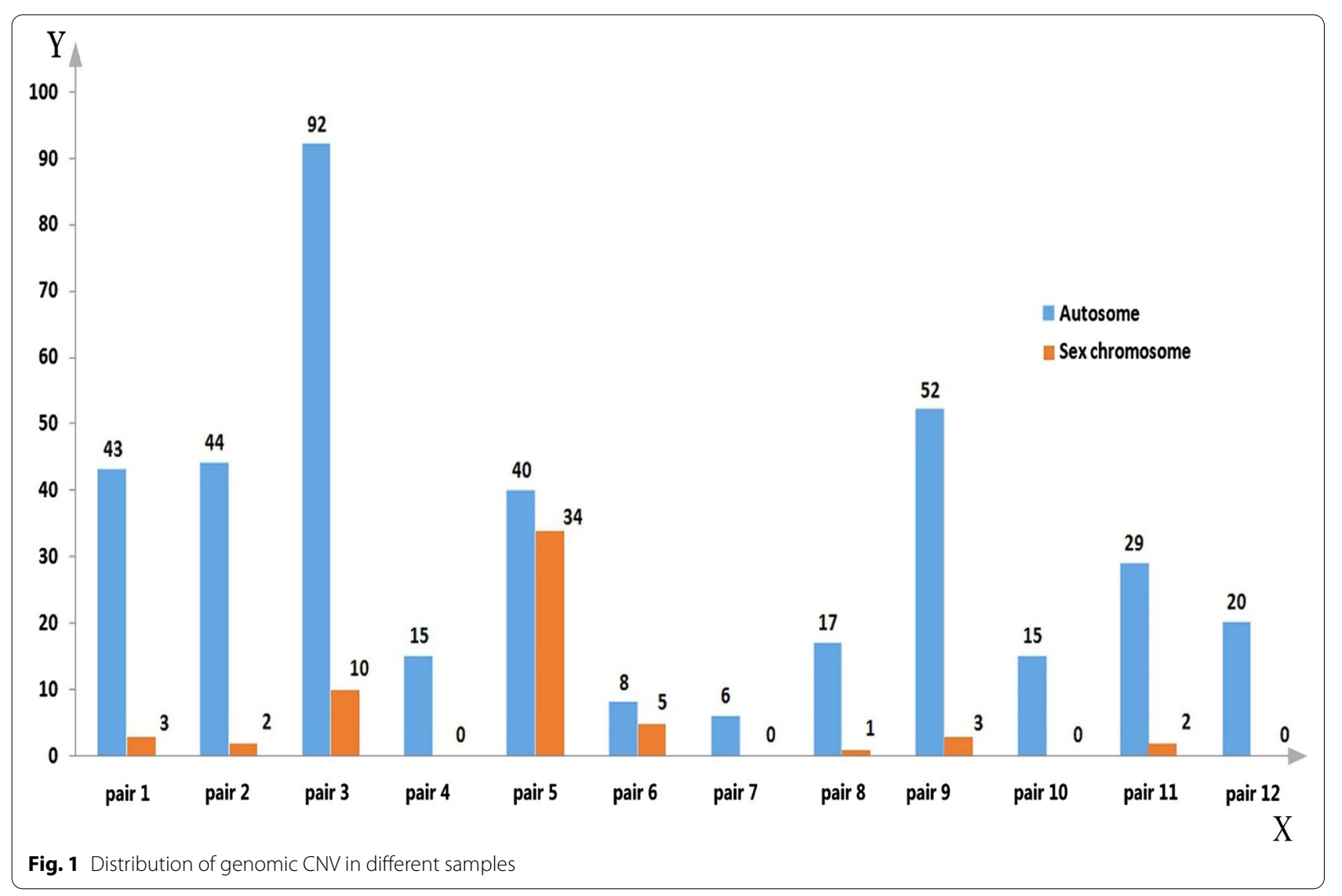

Table 2 CNV detected on autosomes of 12 pairs of samples

\begin{tabular}{|c|c|c|c|c|c|c|}
\hline Sample number & $\begin{array}{l}\text { Gains (CNV- } \\
\text { containing genes) }\end{array}$ & $\begin{array}{l}\text { Losses (CNV- } \\
\text { containing genes) }\end{array}$ & Range of CNV (kb-Mb) & $\begin{array}{l}\text { Total length of } \\
\text { CNV (Mb) }\end{array}$ & $\begin{array}{l}\text { Percentage of } \\
\text { genome length (\%) }\end{array}$ & $\begin{array}{l}\text { Number } \\
\text { of genes }\end{array}$ \\
\hline Pair 1 & $40(26)$ & $3(3)$ & $15.60-2.35$ & 9.91 & 0.17 & 68 \\
\hline Pair 2 & $6(4)$ & $38(32)$ & $7.46-1.93$ & 4.72 & 0.11 & 62 \\
\hline Pair 3 & $5(4)$ & $87(52)$ & $7.46-0.46$ & 8.79 & 0.19 & 88 \\
\hline Pair 4 & $9(8)$ & $6(5)$ & $5.70-1.17$ & 2.68 & 0.05 & 40 \\
\hline Pair 5 & $1(1)$ & $39(31)$ & $8.53-2.23$ & 7.57 & 3.16 & 50 \\
\hline Pair 6 & $4(4)$ & $4(2)$ & $27.55-2.08$ & 3.50 & 0.57 & 22 \\
\hline Pair 7 & $2(2)$ & $4(4)$ & $20.67-0.46$ & 1.03 & 0.60 & 11 \\
\hline Pair 8 & $12(1)$ & $5(3)$ & $10.03-2.35$ & 5.73 & 0.10 & 65 \\
\hline Pair 9 & $6(6)$ & $46(34)$ & $7.46-2.03$ & 7.23 & 0.12 & 86 \\
\hline Pair 10 & $8(7)$ & $7(6)$ & $9.34-2.13$ & 5.49 & 0.09 & 52 \\
\hline Pair 11 & $13(11)$ & $16(14)$ & $17.57-0.85$ & 5.38 & 0.09 & 88 \\
\hline Pair 12 & $11(10)$ & $9(6)$ & $13.20-2.27$ & 7.24 & 0.12 & 82 \\
\hline
\end{tabular}


of NAFLD patients was constructed using R software. A total of 338 autosomal CNVRs were identified, including 275 CNVRs with consistent trends (197 losses and 78 gains) and 63 CNVRS with inconsistent trends (Figs. 2, 3).

The length of the $338 \mathrm{CNVRs}$ ranged from $5.7 \mathrm{~kb}$ to $2.23 \mathrm{Mb}$, with an average size of $117.44 \mathrm{~kb}$. These CNVRs spanned $39.70 \mathrm{Mb}$ of the genome and accounted for $\sim 1.32 \%$ of the genome sequence (Table 3 ). Differences in the number of CNVRs per chromosome were very significant, ranging from 3 for chromosomes 21 and 22 to 36 for chromosome 1 . The scales of CNVRs on each chromosome were also different; CNVRs were densely distributed on chromosomes 7, 8, 15 and 16, covering more than $2 \%$ of genomic sequences, with CNVRs on chromosome 16 covered $3.33 \%$, compared with only $0.11 \%$ for chromosome 20. This demonstrates that the distribution of CNVRs on chromosomes was not uniform.

\section{Gene content of genomic CNVs and identification} of candidate genes associated with NAFLD by functional enrichment.

A total of 1225 genes were annotated in 307 autosomal CNV regions by human genome sequence assembly using Build 37, hg19 (http://www.ncbi.nlm.nih. gov). Functional analysis clustering of these genes was performed using KOBAS gene annotation analysis software, and genes associated with NAFLD were identified using the Pubmed database. Finally, we identified nine CNVRs related to NAFLD (chr3, 57199594-57590187; chr7, 54813380-55274871; chr17, 38688567-38738474; chr19, 56370486-56416408; chr22, 24347959-24390254; chr14, 74001651-74022324; chr12, 57897795-57918452; chr1, 65935075-65959904; chr20, 33470663-33495348) corresponding to genes APPL1, EGFR, CCR7, NLRP4, GSTT1, ACOT1, CHOP, LEPR and ACCS2 (Table 4). By regulating lipid metabolism enzyme activity, adiponectin, insulin signalling, immune cell activity, inflammatory mediator levels and cell phase II response, these genes are closely associated with NAFLD.

\section{Validation of CNV by qPCR}

The results of ACGH showed that 3 out of 12 pairs harboured copy number losses in chr19 (5637048656416408) which contained the NLRP4 gene. Functional enrichment analysis indicated that this gene may participate in inflammatory responses by regulating the levels of inflammatory mediators.

Since inflammatory factors play an important role in the development of NAFLD, quantitative PCR (qPCR)

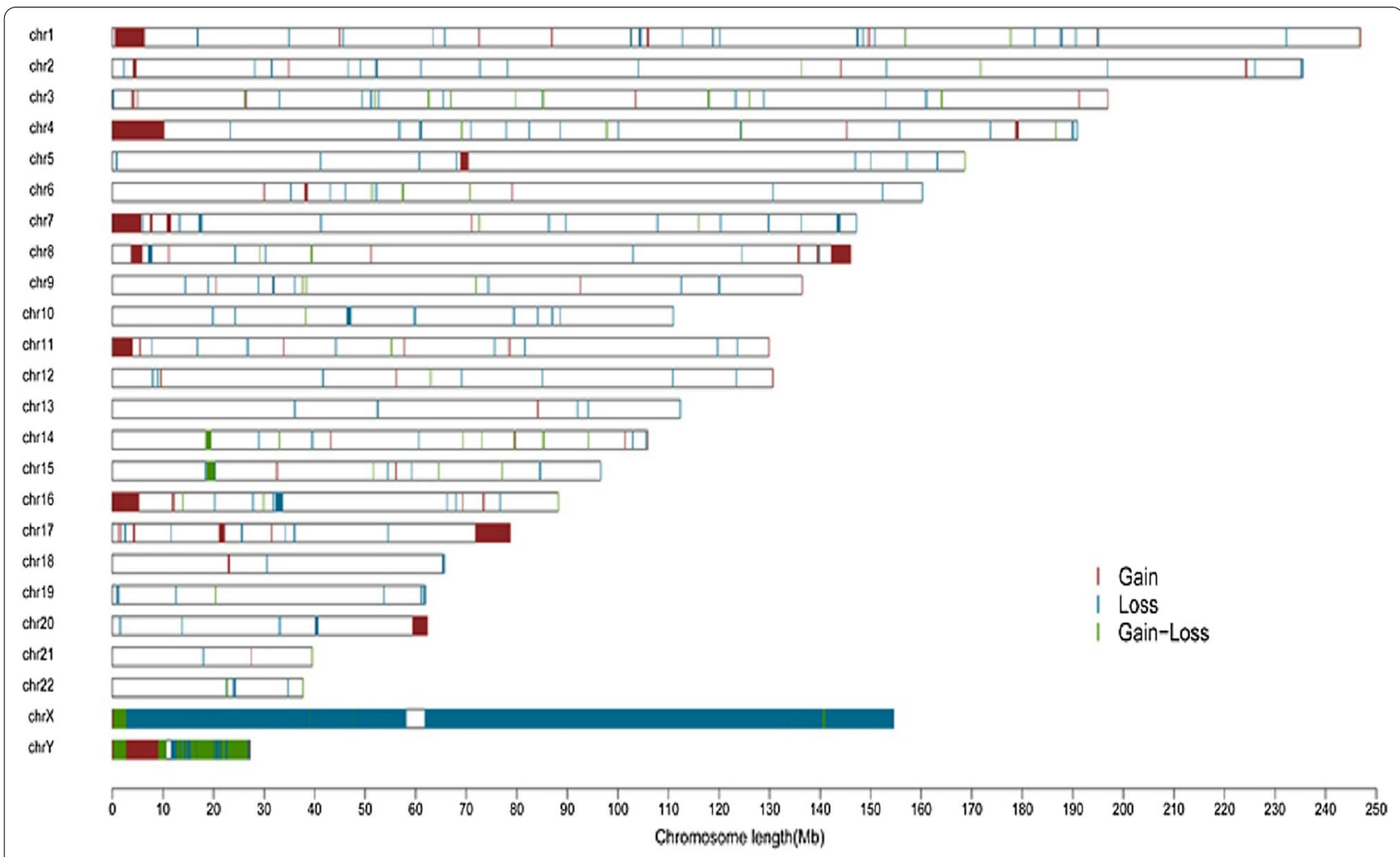

Fig. 2 Distribution of CNVRs in the genome 


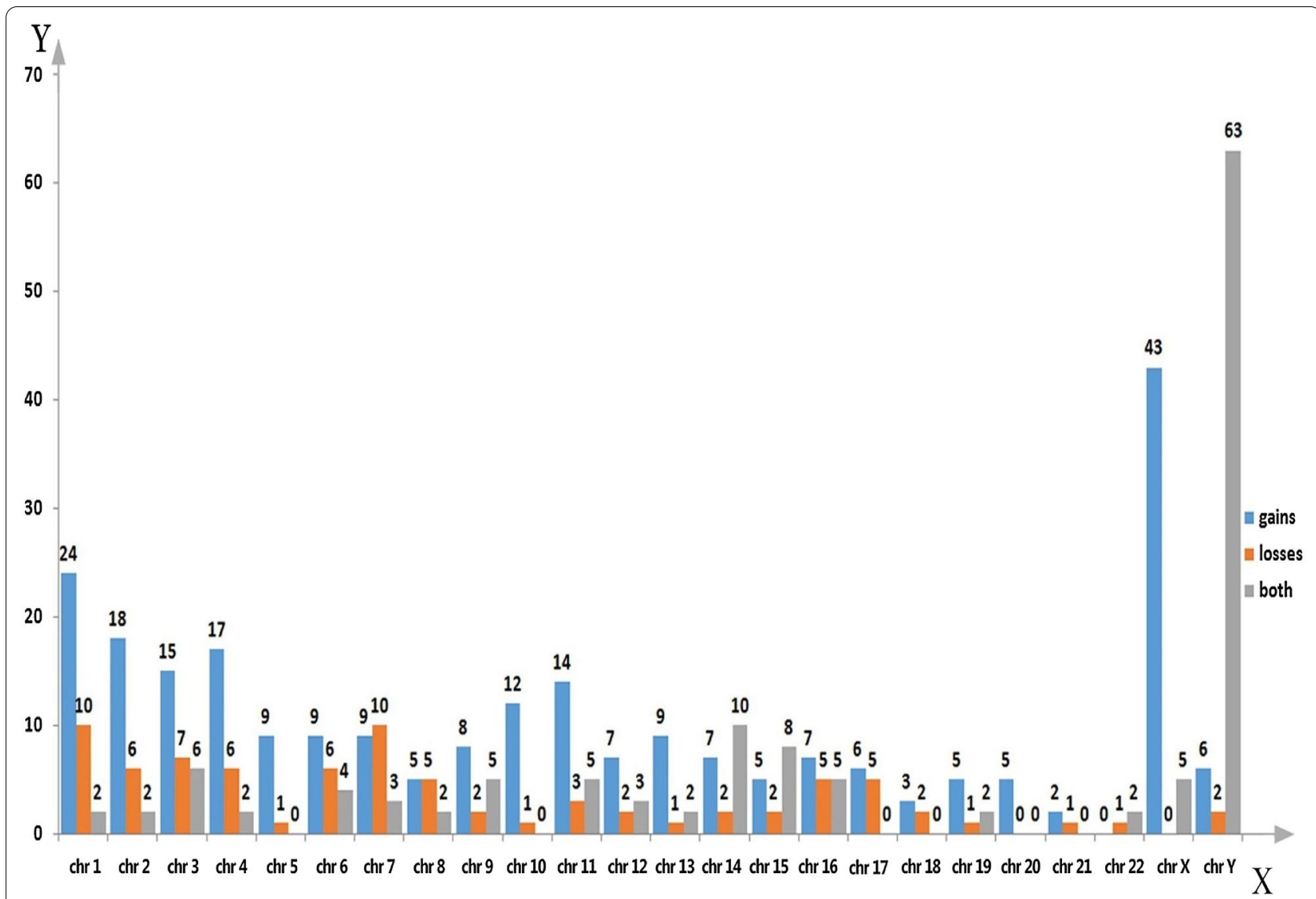

Fig. 3 Genomic CNV trends in different samples

with large sample size was performed to validate this CNV. A total of 557 subjects were included in the study, comprising 297 cases and 260 controls. In case group, the prevalence of obesity and hypertension was significantly higher than that in control group. The level of ALT, AST, FBG and TG were higher in the control group, and the LDL-C was higher in cases (Tables 5, 6).

Copy numbers were calculated using CopyCallerv2.0 software and categorised as losses $(<2)$, normal $(=2)$, and gains $(>2)$. In the present work, the NLRP4 gene copy number distribution ranged from 1 to 2 , with 15 (5.1\%) losses and 282 (94.9\%) normal copy number in cases. There were four (1.5\%) losses and 256 (98.5\%) normal copy number in controls. The distribution of NLRP4 gene copy number variation was statistically significant between the two groups $\left(x^{2}=5.19, p=0.02\right.$; Table 7 , Fig. 4a, b, c). Furthermore, to investigate the relationship between NLRP4 gene copy number variation and the risk of NAFLD, unconditional logistical regression analysis was performed. The results showed that NLRP4 gene copy number deletion was associated with the risk of NAFLD (OR $=3.40,95 \% C I=1.12-10.39)$. After adjustment for confounding factors (gender, age, BMI, blood pressure and diabetes history), the association was still statistically significant $(\mathrm{OR}=4.49,95 \% C I=1.3-15.52$; Table 7).

\section{Discussion}

In this study, we identified 338 autosomal CNV regions, with 1225 genes annotated in 307 of these CNV regions. Through gene enrichment analysis and a review of the existing literature and Pubmed database, we identified nine $\mathrm{CNV}$ regions associated with the development of NAFLD, including chr2 (24347959-24390254, GSTT1), Chr14 (74001651-74022324, ACOT1), chr20 (3347066333495348, ACSS2), Chr7 (54813380-55274871, EGFR), chr1 (65935075-65959904, LEPR), Chr3 (5719959457590187, APPL1), chr12 (57897795-57918452, CHOP), chr19 (56370486-56416408, NLRP4) and chr17 (38688567-38738474, CCR7).

Seven samples exhibited CNV in the region of Chr22 (24347959-24390254) which contained the glutathiones-transferase 1 gene (GSTT1). Six samples displayed copy number gains, and one sample displayed copy number losses. GSTs are a superfamily of proteins that participate in phase II detoxification, which promotes 
Table 3 Distribution of CNVRs in the genome

\begin{tabular}{|c|c|c|c|c|c|c|}
\hline Chr & $\begin{array}{l}\text { No. of CNVRs } \\
\text { (containing genes) }\end{array}$ & Range of CNV (kb-Mb) & Average CNV (kb) & $\begin{array}{l}\text { Total length of } \\
\text { CNV (Mb) }\end{array}$ & $\begin{array}{l}\text { Percentage of } \\
\text { chromosome length (\%) }\end{array}$ & No. Of genes \\
\hline 1 & $36(31)$ & $7.14-0.85$ & 104.26 & 3.75 & 1.5 & 122 \\
\hline 2 & $26(26)$ & $7.46-0.51$ & 105.01 & 2.73 & 1.13 & 44 \\
\hline 3 & $28(24)$ & $10.07-0.39$ & 89 & 2.49 & 1.26 & 50 \\
\hline 4 & $25(25)$ & $9.03-0.58$ & 115.42 & 2.77 & 1.46 & 60 \\
\hline 5 & $10(10)$ & $17.83-1.52$ & 214.13 & 1.93 & 1.06 & 51 \\
\hline 6 & $19(19)$ & $8.95-0.44$ & 59.37 & 1.07 & 0.63 & 34 \\
\hline 7 & $22(19)$ & $8.14-0.69$ & 190.79 & 4.20 & 2.64 & 104 \\
\hline 8 & $12(12)$ & $15.07-2.23$ & 325.54 & 3.58 & 2.47 & 94 \\
\hline 9 & $15(11)$ & $7.63-0.20$ & 52.77 & 0.79 & 0.57 & 14 \\
\hline 10 & $13(11)$ & $13.84-0.73$ & 158.32 & 2.06 & 1.54 & 41 \\
\hline 11 & $22(22)$ & $5.70-0.28$ & 62.75 & 1.32 & 0.98 & 55 \\
\hline 12 & $12(12)$ & $8.68-0.2$ & 46.89 & 0.56 & 0.42 & 29 \\
\hline 13 & $12(8)$ & $17.94-0.35$ & 100.64 & 1.21 & 1.06 & 14 \\
\hline 14 & $19(15)$ & $9.97-0.47$ & 97.33 & 1.85 & 1.73 & 95 \\
\hline 15 & $15(15)$ & $15.60-1.49$ & 181.84 & 2.73 & 2.68 & 113 \\
\hline 16 & $17(14)$ & $25.82-0.8$ & 176.96 & 3.01 & 3.33 & 139 \\
\hline 17 & $11(11)$ & $18.61-0.41$ & 96.05 & 1.06 & 1.27 & 64 \\
\hline 18 & $5(5)$ & $49.08-0.31$ & 125.72 & 0.63 & 0.78 & 13 \\
\hline 19 & $8(8)$ & $16.49-0.42$ & 107.02 & 0.86 & 1.47 & 56 \\
\hline 20 & $5(4)$ & $12-24.69$ & 18.14 & 0.07 & 0.11 & 8 \\
\hline 21 & $3(2)$ & $0.98-0.11$ & 41.48 & 0.12 & 0.26 & 4 \\
\hline 22 & $3(3)$ & $26.37-0.29$ & 119.47 & 0.36 & 0.71 & 21 \\
\hline$x$ & $48(40)$ & $17.85-31.52$ & 3143.22 & 150.87 & 96.69 & 2357 \\
\hline y & $71(62)$ & $12.82-3.96$ & 357.95 & 25.41 & 44.40 & 506 \\
\hline
\end{tabular}

Table 4 CNV region associated with NAFLD

\begin{tabular}{|c|c|c|c|c|c|}
\hline Region & Gene & $\begin{array}{l}\text { Sample } \\
\text { frequency } \\
\text { (gains, losses) }\end{array}$ & Function & GO item & Relevant study ( DOI) \\
\hline Chr3:57199594-57590187 & APPL1 & $1(0,1)$ & Adiponectin, insulin signalling & GO:0008286 & 10.1371/journal.pone.0071391 \\
\hline Chr7:54813380-55274871 & EGFR & $1(0,1)$ & $\begin{array}{l}\text { Tyrosine phosphorylation regulates cell } \\
\text { proliferation }\end{array}$ & GO:0000165 & $\begin{array}{l}\text { 10.3969/j.issn.1674-4136.2017.05.016; } \\
\text { 10.1016/j.bbadis.2017.10.016 }\end{array}$ \\
\hline Chr17:38688567-38738474 & CCR7 & $2(0,2)$ & Participates inflammatory response & GO:0007249 & $\begin{array}{l}\text { 10.1038/ijo.2017.200; 10.3870/j. } \\
\text { issn.1672-0741.2017.06.011 }\end{array}$ \\
\hline Chr19:56370486-56416408 & NLRP4 & $3(0,3)$ & $\begin{array}{l}\text { Inhibition of type I interferon and } \\
\text { NF-KB inflammatory signalling }\end{array}$ & GO:0032479 & $\begin{array}{l}\text { 10.1186/s12876-014-0208-8; 10.1074, } \\
\text { jbc.M200446200 }\end{array}$ \\
\hline Chr22:24347959-24390254 & GSTT1 & $7(6,1)$ & $\begin{array}{l}\text { Participates in the phase II reaction and } \\
\text { promote toxic metabolism }\end{array}$ & GO:0004602 & 10.1016/j.clinre.2011.01.015 \\
\hline Chr14:74001651-74022324 & ACOT1 & $2(1,1)$ & $\begin{array}{l}\text { Regulation of lipid metabolism } \\
\text { enzyme activity }\end{array}$ & GO:0071616 & $\begin{array}{l}\text { 10.2337/db16-1519; 10.1194/jlr. } \\
\text { M081455 }\end{array}$ \\
\hline Chr12:57897795-57918452 & $\mathrm{CHOP}$ & $1(1,0)$ & $\begin{array}{l}\text { Participates in endoplasmic reticulum } \\
\text { stress and promote apoptosis }\end{array}$ & GO:0036488 & $\begin{array}{l}\text { 10.1038/labinvest.2016.61; 10.1016/j. } \\
\text { ejps.2011.02.005 }\end{array}$ \\
\hline Chr1:65935075-65959904 & LEPR & $1(0,1)$ & Regulates leptin signalling & GO:0038021 & $\begin{array}{l}\text { 10.2174/15665240186661807051 } \\
\text { 10412; } 10.3870 / \text { /cxh.j.issn.1005- } \\
\text { 541X.2011.03.07 }\end{array}$ \\
\hline Chr20:33470663-33495348 & ACSS2 & $1(0,1)$ & $\begin{array}{l}\text { Regulation of lipid metabolism } \\
\text { enzyme activity }\end{array}$ & GO:0006085 & $\begin{array}{l}\text { 10.1073/pnas.1806635115; } 10.1002 / \\
\text { jcp.25954 }\end{array}$ \\
\hline
\end{tabular}


Table 5 Baseline characteristics of the study population

\begin{tabular}{|c|c|c|c|c|}
\hline & Cases (\%) & Controls (\%) & $x^{2} / Z$ & pvalue \\
\hline \multicolumn{5}{|l|}{ Gender } \\
\hline Male & $209(70.4)$ & $185(71.2)$ & 0.04 & 0.84 \\
\hline Female & $88(29.6)$ & $75(28.8)$ & & \\
\hline Age (years) & $45.66 \pm 11.764$ & $45.48 \pm 11.804$ & -0.16 & 0.87 \\
\hline \multicolumn{5}{|l|}{ Education } \\
\hline Primary education & $63(21.2)$ & $55(21.2)$ & 1.97 & 0.37 \\
\hline $\begin{array}{l}\text { Secondary educa- } \\
\text { tion }\end{array}$ & $88(29.6)$ & $64(24.6)$ & & \\
\hline Bachelor degree & $146(49.2)$ & $141(54.2)$ & & \\
\hline \multicolumn{5}{|l|}{ Marital status } \\
\hline Single & $31(10.4)$ & $36(13.8)$ & 1.52 & 0.22 \\
\hline Married or divorced & $266(89.6)$ & $224(86.2)$ & & \\
\hline \multicolumn{5}{|l|}{ Income (¥) } \\
\hline$<1000$ & $16(5.4)$ & $15(5.8)$ & 0.36 & 0.83 \\
\hline $1000-2000$ & $83(27.9)$ & $78(30)$ & & \\
\hline$\geq 2000$ & $198(66.7)$ & $167(64.2)$ & & \\
\hline \multicolumn{5}{|l|}{$B M I\left(\mathrm{~kg} / \mathrm{m}^{2}\right)$} \\
\hline$<18.5$ & $2(0.7)$ & $9(3.5)$ & 117.95 & $<0.001$ \\
\hline $18.5-23.9$ & $99(33.3)$ & $193(74.2)$ & & \\
\hline $24.0-27.9$ & $149(50.2)$ & $57(21.9)$ & & \\
\hline$\geq 28$ & $47(15.8)$ & $1(0.4)$ & & \\
\hline \multicolumn{5}{|l|}{$\begin{array}{l}\text { Blood pressure }(\mathrm{mm} / \\
\mathrm{Hg})\end{array}$} \\
\hline$<140 / 90$ & $211(71)$ & $211(81.2)$ & 7.72 & 0.01 \\
\hline$\geq 140 / 90$ & $86(29)$ & $49(18.8)$ & & \\
\hline \multicolumn{5}{|l|}{ Diabetes } \\
\hline Yes & $12(4)$ & $7(97.3)$ & 0.77 & 0.38 \\
\hline No & $285(96)$ & $253(2.7)$ & & \\
\hline \multicolumn{5}{|l|}{ Tea consumption } \\
\hline No & $112(37.7)$ & $109(41.9)$ & 1.03 & 0.311 \\
\hline Yes & $185(62.3)$ & $151(58.1)$ & & \\
\hline \multicolumn{5}{|l|}{ Smoking habit } \\
\hline No & $220(74.1)$ & $197(75.8)$ & 0.21 & 0.65 \\
\hline Yes & 77 (25.9) & $63(24.2)$ & & \\
\hline
\end{tabular}

Bold indicates that the analysis result is statistically significant

Table 6 Clinical characteristics of NAFLD patients and normal controls

\begin{tabular}{lccll}
\hline Characteristics & Cases (\%) & Controls (\%) & Z & pvalue \\
\hline ALT (IU/l) & $27(19-38)$ & $19(14-27)$ & -7.91 & $<\mathbf{0 . 0 0 1}$ \\
AST (IU/l) & $23(20-28)$ & $20(18-24)$ & -5.59 & $<\mathbf{0 . 0 0 1}$ \\
GLU (mmol/l) & $5.34(4.97-5.87)$ & $5.19(4.91-5.52)$ & -3.34 & $\mathbf{0 . 0 0 1}$ \\
TC $(\mathrm{mmol} / \mathrm{l})$ & $5.09(4.49-5.65)$ & $5.03(4.56-5.45)$ & -0.94 & 0.35 \\
TG $(\mathrm{mmol} / \mathrm{l})$ & $1.77(1.26-2.51)$ & $1.18(0.87-1.55)$ & -9.50 & $<\mathbf{0 . 0 0 1}$ \\
HDL (mmol/l) & $3.13(2.53-3.75)$ & $3.19(2.687-3.56)$ & -0.49 & 0.62 \\
LDL (mmol/l) & $1.21(1.06-1.37)$ & $1.35(1.20-1.47)$ & -6.70 & $<\mathbf{0 . 0 0 1}$
\end{tabular}

Bold indicates that the analysis result is statistically significant

AST serum aspartate aminotransferase, $A L T$ alanine aminotransferase, GGT gamma-glutamyltranspeptidase, $F B G$ serum fasting glucose, $T C$ total cholesterol $T G$ triglycerides, LDL-C low-density lipoprotein, HDL-C high-density lipoprotein the elimination of toxic metabolites and reduces oxidative damage by catalysing the conjugated binding of glutathione to electrophilic substances (such as peroxides, superoxide ions, lipid peroxides, etc.). Studies have shown the GSTT1 gene polymorphism is closely related to the risk of metabolic diseases such as coronary heart disease [18] and diabetes [19]. However, one study targeted at Southeast Iran showed that genetic polymorphism of GSTM1 and GSTP1, but not GSTT1, was associated with NAFLD [20].

The CNV regions Chr14:74001651-74022324 and chr20:33470663-33495348 contained the genes acylCoA thioesterase 1 (ACOT1) and acyl-CoA synthetase short-chain family member 2 (ACSS2), both of which are related to lipid metabolism. ACOT1 is a key cytosolic enzyme that participates in fatty acid (FA) metabolism by catalysing the conversion of acyl-CoAs to FAs and coenzyme $\mathrm{A}$, and it is also believed to be a target gene of PPAR $\alpha$ [21-23]. Previous studies revealed that overexpression of ACOT1 reduces the availability of excess long-chain acyl-CoAs for $\beta$-oxidation, and represses PPAR $\alpha$ signalling pathways to reverse altered substrate metabolism and attenuate increased oxidative stress, mitochondrial dysfunction, and cardiac inefficiency in diabetic hearts [24]. In mice fed a high fat diet, Acot1 knockdown elicits a robust induction of inflammatory and oxidative stress markers, and increased ROS levels. Thus, when a high fat diet induces steatosis, ACOT1 protects against inflammation and oxidative stress that lead to fibrosis [25].

ACSS2 plays a key role in lipogenesis by converting acetate to acetyl-CoA for lipogenesis [26]. A recent study showed that knockdown of ACSS2 increased the invasion and migration abilities of HCC cells, which plays an important role in the prognosis of patients with $\mathrm{HCC}$ [27]. Another study also found that mRNA levels of genes associated with de novo fatty acid synthesis, triacylglycerol synthesis, lipid droplet formation and fatty acid oxidation were downregulated after ACSS2 knockdown [28]. In diet-induced obese mice, lack of ACSS2 results in a significant reduction in body weight and hepatic steatosis [29].

The CNV region chr1:65935075-65959904 contains the gene encoding leptin receptor (LEPR) that binds to leptin in target tissues. Intravenous administration of leptin reduces appetite, while its deficiency increases food intake. Furthermore, leptin can modulate insulin secretion and action through LEPR, and polymorphism of LEPR has been linked to NAFLD [30].

The CNV region Chr3:57199594-57590187 contains genes encoding Adaptor protein and $\mathrm{PH}$ domain- and leucine zipper-containing 1 (APPL1). APPL1 is the first identified adaptor protein that interacts directly with 
Table 7 Relationship between NLRP4 gene copy number variation and the risk of NAFLD

\begin{tabular}{|c|c|c|c|c|c|}
\hline \multirow[t]{2}{*}{ Group } & \multirow[t]{2}{*}{ One copy } & \multirow[t]{2}{*}{ Two copies } & \multirow[t]{2}{*}{$x^{2}(p)$} & \multicolumn{2}{|l|}{ OR $(95 \% C l)$} \\
\hline & & & & Model 1 & Model 2 \\
\hline Cases & $15(5.1)$ & $282(94.9)$ & $5.19(0.02)$ & $3.40(1.12-10.39)$ & $4.49(1.3-15.52)$ \\
\hline Controls & $4(1.5)$ & 256 (98.5) & & & \\
\hline
\end{tabular}

Model 1: unadjusted model

Model 2: adjusted for gender, age, blood pressure, BMI, Diabetes history

adiponectin receptors. Adiponectin is a peptide secreted by adipocytes that plays an important role in regulating glucose and lipid metabolism, and controlling inflammation in insulin-sensitive tissues. Studies have linked low adiponectin levels to NAFLD. Adiponectin signalling through APPL1 is necessary to exert its anti-inflammatory and cytoprotective effects on endothelial cells. APPL1 also functions in insulin signalling pathways, it is an important mediator of adiponectin-dependent insulin sensitisation in skeletal muscle, and it acts as a mediator of other signalling pathways by interacting directly with membrane receptors or signalling proteins. Thus, APPL1 plays critical roles in cell proliferation, apoptosis, cell survival, endosomal trafficking and chromatin remodelling [31]. Polymorphism of APPL1 has been associated with NAFLD [32].

The CNV region chr19:56370486-56416408 contains the gene encoding NLRP4, a negative regulator of proinflammatory cytokines that is associated with inactivation of IKKa/NF-kB [33]. A previous study demonstrated that NLRP4 attenuates the inflammation response by reducing the expression of transforming growth factor- $\beta 1$ (TGF- $\beta 1$ ), tumour necrosis factor- $\alpha$ (TNF- $\alpha$ ), interleukin-1 $\beta$ (IL-1 $\beta$ ), IL-18 and IL-6 in fructose-treated cardiac cells [34]. In visceral adipose tissue from patients with pericellular fibrosis, NLRP4 mRNA levels were significantly lower than in those without pericellular fibrosis, and the NLRP 4 gene is significantly downregulated in adipose tissue from NASH patients [35].

The CNV region chr17:38688567-38738474 contains the gene encoding $\mathrm{C}-\mathrm{C}$ chemokine receptor 7 (CCR7), which is primarily expressed in immune cells. Studies found that CCR7 deficiency leads to the development of multi-organ autoimmunity [36], chronic renal disease [37] and autoimmune diabetes [38]. A recent study showed that infiltration of CCR7-expressing cells in adipose tissue is associated with insulin resistance in obesity [39]. The absence of CCR7 decreases IL-10-producing iNKT cells in fatty liver, and exacerbates NAFLD [40].

The CNV region Chr7:54813380-55274871 contains the gene encoding epidermal growth factor receptor (EGFR), a receptor tyrosine kinase expressed in activated hepatic stellate cells (HSCs) that is associated with the development of liver fibrosis. One study indicated that depressing the phosphorylation of EGFR can reduce the number of activated HSCs [41]. Another study suggested that EGFR is phosphorylated in liver tissues in a high-fat diet-induced murine model of NAFLD. Inhibition of EGFR prevents diet-induced lipid accumulation, oxidative stress, HSC activation and matrix deposition [42].

The CNV region chr12:57897795-57918452 contains the gene encoding CCAAT/enhancer-binding protein $(\mathrm{C} / \mathrm{EBP})$ homologous protein $(\mathrm{CHOP})$, a major transcriptional regulator of endoplasmic reticulum (ER) stress-mediated apoptosis that is implicated in lipotoxicity-induced ER stress and hepatocyte apoptosis in NAFLD [43]. A previous study found that CHOP knockout (CHOP-/-) mice fed a high-fat diet developed more severe histological NASH features than wildtype controls; The severity of NASH in high-fat diet-fed $\mathrm{CHOP}-/-$ mice correlated with a significant decrease in peroxisomal $\beta$-oxidation, increased denovo lipogenesis, and ER stress-mediated hepatocyte apoptosis [44]. These findings indicate that $\mathrm{CHOP}$ protects hepatocytes from ER stress, and plays a significant role in the mechanism of liraglutide-mediated protection against NASH pathogenesis.

The use of ACGH technology allows CNV discovery at high resolution, and hence confidence in CNV detection. Therefore, this approach may be used to identify noninvasive biomarkers with potential for the pathological evaluation of NAFLD. However, as more comprehensive studies are still ongoing, genes known to be related to NAFLD will be investigated, and our preliminary results remain to be substantiated by studies on larger patient groups. In addition, functional studies on genes residing within these loci are needed to fully characterise the functions of genes and their relationships with NAFLD.

\section{Conclusions}

In case group, the copy number deletion of NLRP4 gene in the Chr19:56370486-56416408 region was higher than that in control group. The copy number deletion of this gene was related to the risk of NAFLD. 


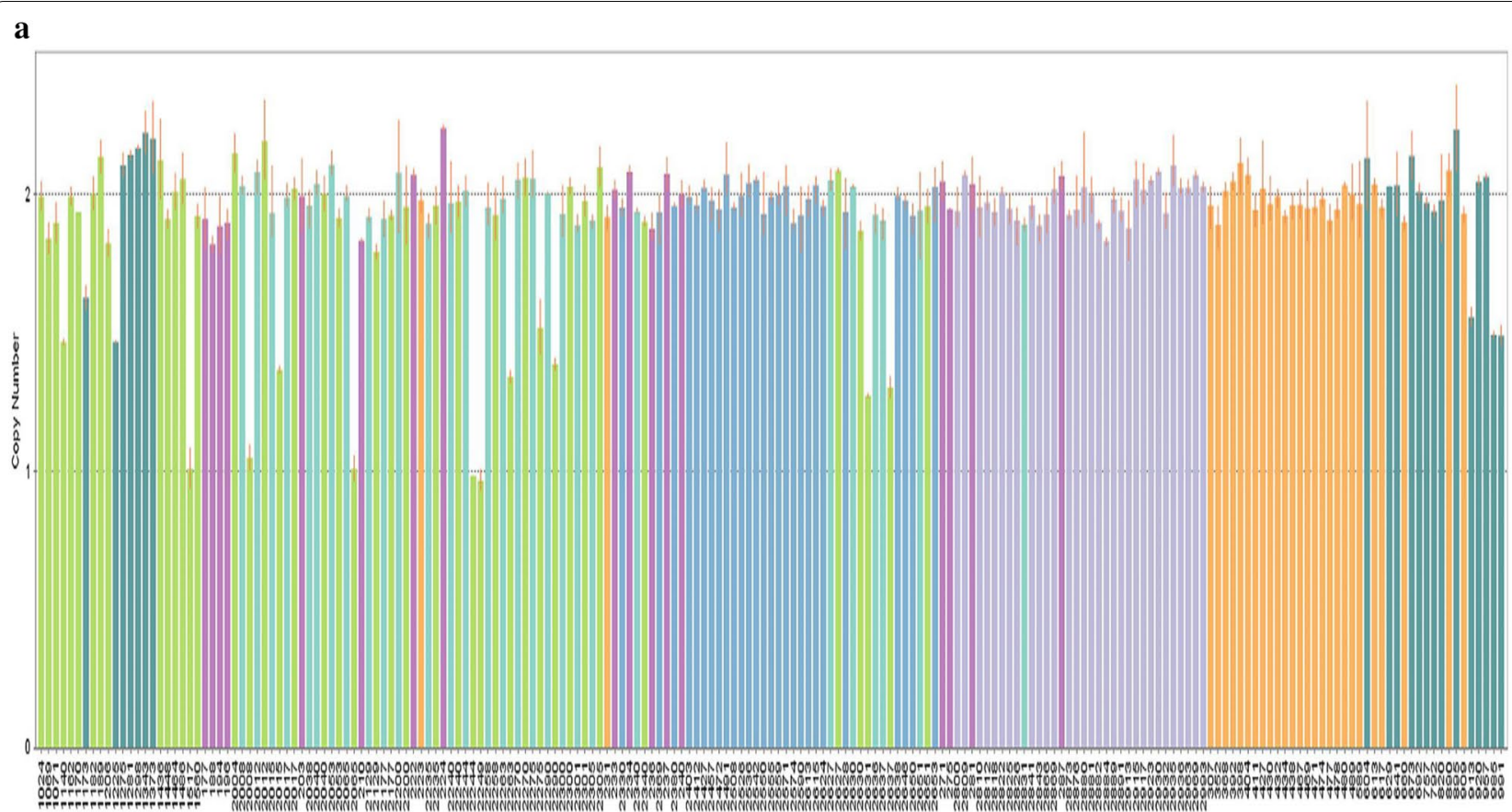

b

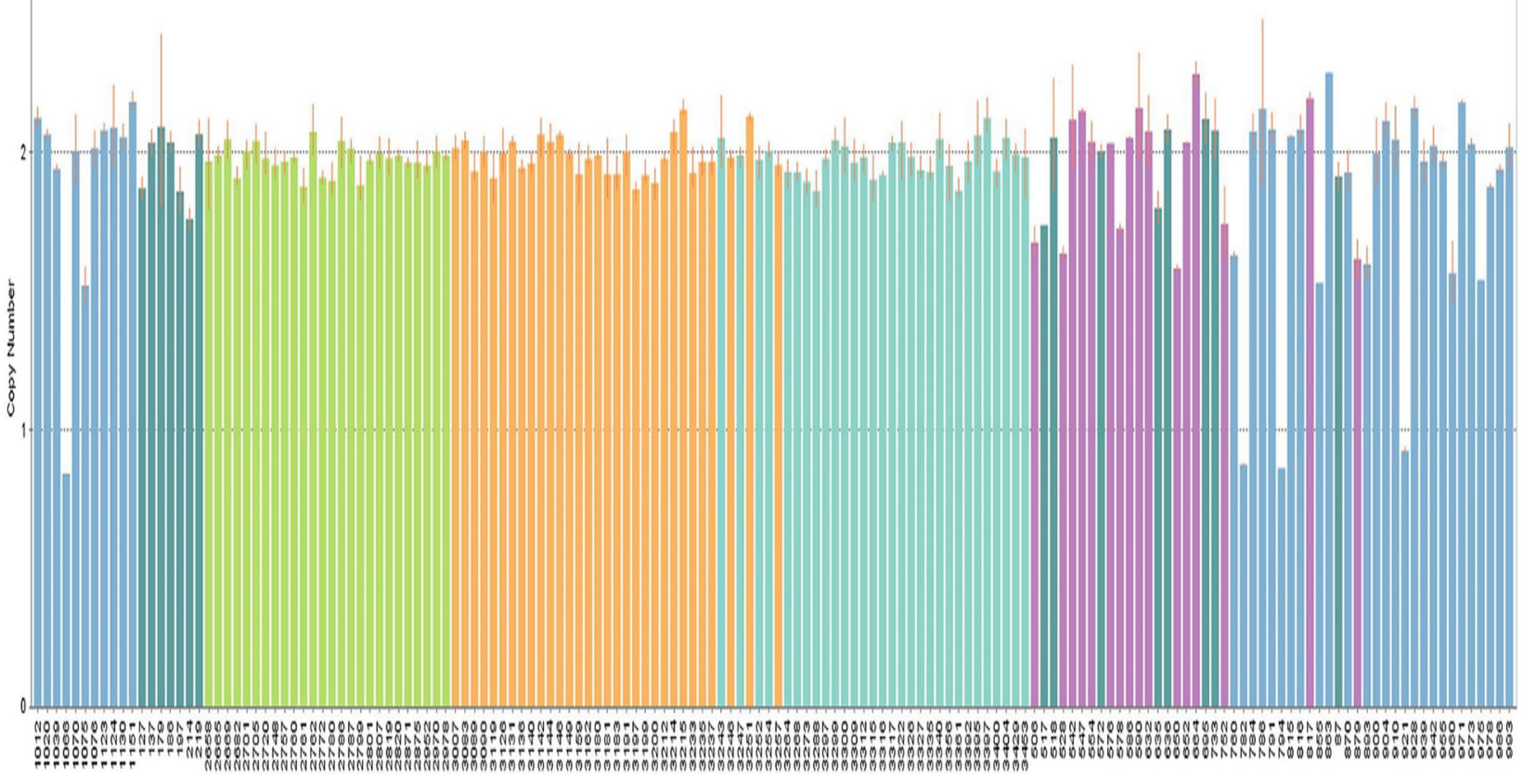

Fig. 4 Copy number distribution of the NLRP4 gene (Because the copy number analysis software (copy caller) has a limit on the number of samples in each analysis, all the experimental samples were randomly grouped and analyzed in three times, and finally three figures were obtained: Figure a, $b, c)$ 


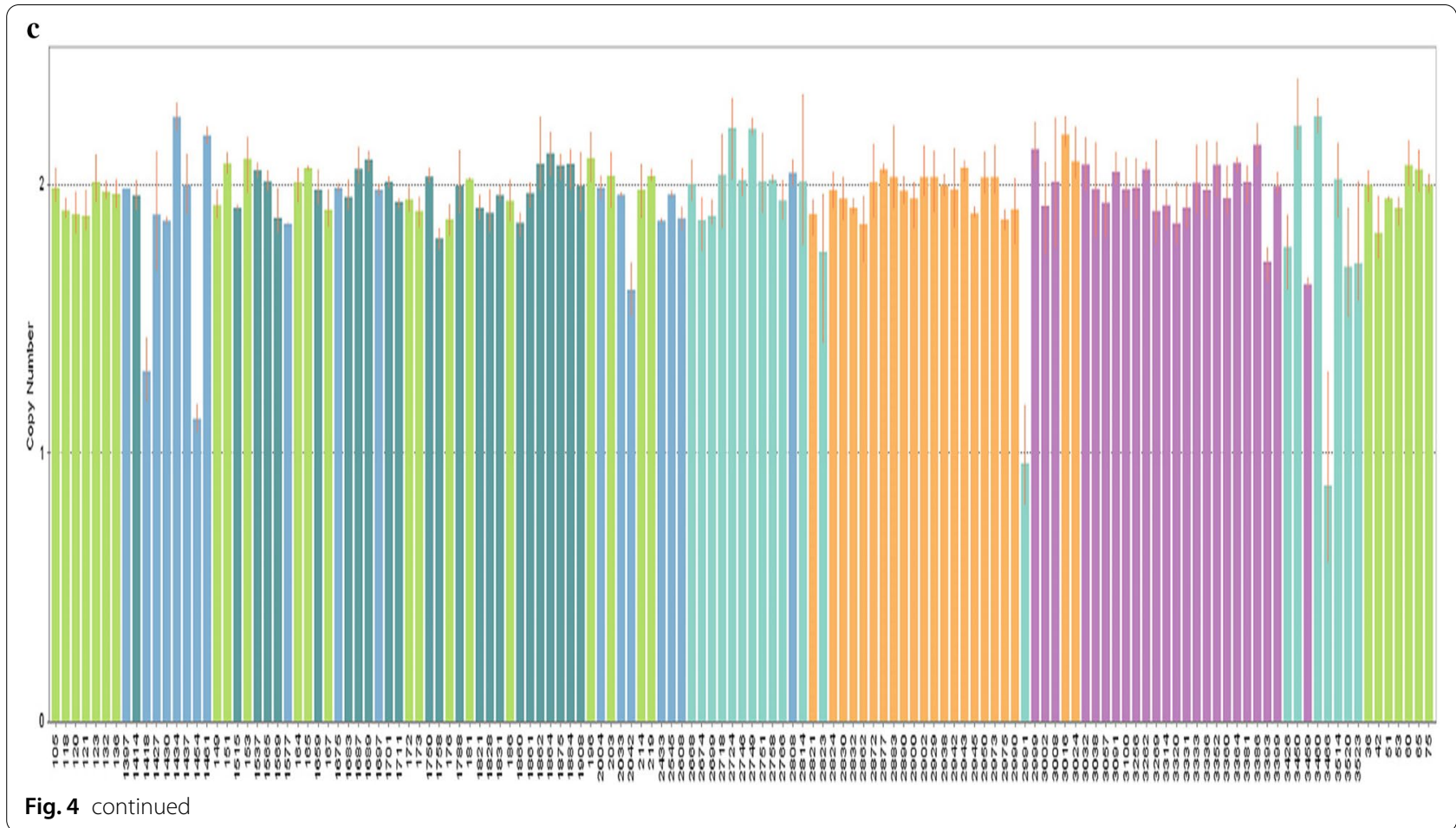

\section{Abbreviations}

ACGH: Array-based comparative genomic hybridization; AST: Asparagine aminnotransferase; ALT: Alanine aminotransferase; BMI: Body mass index; CNV: Copy number variation; FBG: Fasting blood glucose; GGT: $y$-Glutamyl transferase; HDL-C: HDL cholesterol; IR: Insulin resistance; LDL-C: LDL cholesterol; NAFLD: Non-alcoholic fatty liver disease; NLRP4: NLR family pyrin domain containing 4; OR: Odds ratio; TC: Total cholesterol; TG: Triglycerides.

\section{Supplementary Information}

The online version contains supplementary material available at https://doi. org/10.1186/s12876-021-01750-4.

Additional file 1. CNV detected on sex chromosomes of 12 pairs of samples.

\section{Acknowledgements}

We thank all study participants for their cooperation. We also thank our staff for recruiting subjects, and for their technical assistance.

\section{Authors' contributions}

$Y L$ and JZ are joint first authors. XEP obtained funding and designed the study. $\mathrm{HP}, \mathrm{XC}, \mathrm{HL}$ and $\mathrm{QH}$ collected the data. XP and $\mathrm{YL}$ were involved in data verification and analysis. YL and JZ drafted the manuscript. ZH, YW and XEP contributed to the interpretation of the results and critical revision of the manuscript for important intellectual content and approved the final version of the manuscript. All authors read and approved the final manuscript.

\section{Funding}

This work was supported by the National Natural Science Foundation of China [Grant Nos.: 81473047], Joint funds for the innovation of science and technology, Fujian province [Grant Nos.: 2017 Y9104 and 2016Y9035], the Natural Science Foundation of Fujian province [Grant No.: 2019J01306]. They provide financial support for our research.

\section{Availability of data and materials}

All data generated or analyzed during this study are included in this published article or are available from the corresponding author on reasonable request.

\section{Declarations}

\section{Ethics approval and consent to participate}

This study was approved by the Medical Ethical committee of School of Fujian Medical University (Fujian, China, ethics number 2014096).

\section{Consent for publication}

Informed consent was obtained in writing by all participates.

\section{Competing interests}

The authors declare that they have no competing interests.

Received: 7 October 2020 Accepted: 30 March 2021

Published online: 14 April 2021

\section{References}

1. Rinella ME. Nonalcoholic fatty liver disease: a systematic review. JAMA. 2015;313(22):2263-73.

2. Ortiz-Lopez C, Lomonaco R, Orsak B, Finch J, Chang Z, Kochunov V, Hardies J, Cusi K. Prevalence of prediabetes and diabetes and metabolic profile of patients with nonalcoholic fatty liver disease (NAFLD). Diabetes Care. 2012;35(4):873-8.

3. Motamed N, Rabiee B, Poustchi H, Dehestani B, Hemasi G, Khonsari M, Maadi M, Saeedian F, Zamani F. Non-alcoholic fatty liver disease (NAFLD) and 10-year risk of cardiovascular diseases. Clin Res HepatolGastroenterol. 2017:41(1):31-8.

4. Gaggini M, Morelli M, Buzzigoli E, DeFronzo R, Bugianesi E, Gastaldelli A. Non-alcoholic fatty liver disease (NAFLD) and its connection with insulin resistance, dyslipidemia, atherosclerosis and coronary heart disease. Nutrients. 2013:5(5):1544-60. 
5. Namjou B, Lingren T, Huang Y, Parameswaran S, Cobb B, Stanaway I, Connolly J, Mentch F, Benoit B, Niu X, et al. GWAS and enrichment analyses of non-alcoholic fatty liver disease identify new trait-associated genes and pathways across eMERGE Network. BMC Med. 2019;17(1):135.

6. Liu J, Xing J, Wang B, Wei C, Yang R, Zhu Y, Qiu H. Correlation between adiponectin gene rs1501299 polymorphism and nonalcoholic fatty liver disease susceptibility: a systematic review and meta-analysis. Med SciMonitInt Med J Exp Clin Res. 2019;25:1078-86.

7. Kovalic A, Banerjee P, Tran Q, Singal A, Satapathy S. Genetic and epigenetic culprits in the pathogenesis of nonalcoholic fatty liver disease. J Clin ExpHepatol. 2018;8(4):390-402.

8. Feuk L, Carson AR, Scherer SW. Structural variation in the human genome. Nat Rev Genet. 2006;7(2):85-97.

9. Sekine M, Makino T. Inference of causative genes for alzheimer's disease due to dosage imbalance. Mol Biol Evol. 2017;34(9):2396-407.

10. Mahne AC, Carr JA, Bardien S, Schutte CM. Clinical findings and genetic screening for copy number variation mutations in a cohort of South African patients with Parkinson's disease. South Afr Med J. 2016;106(6):623.

11. Sriretnakumar V, Zai CC, Wasim S, Barsanti-Innes B, Kennedy JL, So J. Copy number variant syndromes are frequent in schizophrenia: progressing towards a CNV-schizophrenia model. Schizophr Res. 2019;209:171-8.

12. Lu X, Ye K, Zou K, Chen J. Identification of copy number variationdriven genes for liver cancer via bioinformatics analysis. Oncol Rep. 2014;32(5):1845-52.

13. Hashimoto T, Osoegawa A, Takumi Y, Abe M, Kobayashi R, Miyawaki M, Takeuchi H, Okamoto T, Sugio K. Intratumoral heterogeneity of copy number variation in lung cancer harboring L858R via immunohistochemical heterogeneous staining. Lung Cancer (Amsterdam, Netherlands). 2018;124:241-7.

14. Farrell GC, Shivakumar C, Lau GKK, Sollano JD. Guidelines for the assessment and management of non-alcoholic fatty liver disease in the Asia-Pacific region: executive summary. J GastroenterolHepatol. 2010;22(6):775-7.

15. Saverymuttu S, Joseph A, Maxwell J. Ultrasound scanning in the detection of hepatic fibrosis and steatosis. Br Med J (Clin Res Ed). 1986;292(6512):13-5.

16. De A. N C: Drug-induced hepatic steatosis. Semin Liver Dis. 2014;34(2):205-14.

17. Xie C, Mao X, Huang J, Ding Y, Wu J, Dong S, Kong L, Gao G, Li CY, Wei L. KOBAS 2.0: a web server for annotation and identification of enriched pathways and diseases. Nucleic Acids Res. 2011;39(Web Server issue):W316-322.

18. Song Y, Shan Z, Luo C, Kang C, Yang Y, He P, Li S, Chen L, Jiang X, Liu L. Glutathione S-transferase T1 (GSTT1) null polymorphism, smoking, and their interaction in coronary heart disease: a comprehensive meta-analysis. Heart Lung Circ. 2017;26(4):362-70.

19. Petrovič D, Peterlin B. GSTM1-null and GSTT1-null genotypes are associated with essential arterial hypertension in patients with type 2 diabetes. Clin Biochem. 2014:47(7-8):574-7.

20. Hashemi M, Eskandari-Nasab E, Fazaeli A, Bahari A, Hashemzehi NA, Shafieipour S, Taheri M, Moazeni-Roodi A, Zakeri Z, Bakhshipour A, et al. Association of genetic polymorphisms of glutathione-S-transferase genes (GSTT1, GSTM1, and GSTP1) and susceptibility to nonalcoholic fatty liver disease in Zahedan, Southeast Iran. DNA Cell Biol. 2012;31(5):672-7.

21. Dongol B, Shah Y, Kim I, Gonzalez F, Hunt M. The acyl-CoA thioesterase I is regulated by PPARalpha and HNF4alpha via a distal response element in the promoter. J Lipid Res. 2007:48(8):1781-91.

22. Hunt M, Alexson S. The role Acyl-CoA thioesterases play in mediating intracellular lipid metabolism. Prog Lipid Res. 2002;41(2):99-130.

23. Hunt M, Yamada J, Maltais L, Wright M, Podesta E, Alexson S. A revised nomenclature for mammalian acyl-CoA thioesterases/hydrolases. J Lipid Res. 2005;46(9):2029-32.

24. Yang S, Chen C, Wang H, Rao X, Wang F, Duan Q, Chen F, Long G, Gong W, Zou MH, et al. Protective effects of Acyl-coA thioesterase 1 on diabetic heart via PPARa/PGC1 a signaling. PLoS ONE. 2012;7(11):e50376.

25 Franklin MP, Sathyanarayan A, Mashek DG. Acyl-CoA thioesterase 1 (ACOT1) regulates PPARa to couple fatty acid flux with oxidative capacity during fasting. Diabetes. 2017;66(8):2112-23.

26. Schug ZT, Peck B, Jones DT, Zhang Q, Grosskurth S, Alam IS, Goodwin LM, Smethurst E, Mason S, Blyth K, et al. Acetyl-CoA synthetase 2 promotes acetate utilization and maintains cancer cell growth under metabolic stress. Cancer Cell. 2015;27(1):57-71.
27. Sun L, Kong Y, Cao M, Zhou H, Li H, Cui Y, Fang F, Zhang W, Li J, Zhu X, et al. Decreased expression of acetyl-CoA synthase 2 promotes metastasis and predicts poor prognosis in hepatocellular carcinoma. Cancer Sci. 2017;108(7):1338-46.

28. Xu H, Luo J, Ma G, Zhang X, Yao D, Li M, Loor JJ. Acyl-CoA synthetase short-chain family member 2 (ACSS2) is regulated by SREBP-1 and plays a role in fatty acid synthesis in caprine mammary epithelial cells. J Cell Physiol. 2018;233(2):1005-16.

29. Huang Z, Zhang M, Plec AA, Estill SJ, Cai L, Repa JJ, McKnight SL, Tu BP ACSS2 promotes systemic fat storage and utilization through selective regulation of genes involved in lipid metabolism. ProcNatlAcadSci USA. 2018;115(40):E9499-506.

30. An BQ, Lu LL, Yuan C, Xin YN, Xuan SY. Leptin receptor gene polymorphisms and the risk of non-alcoholic fatty liver disease and coronary atherosclerosis in the Chinese Han Population. Hepat Mon. 2016;16(4):e35055.

31. Fisman EZ, Tenenbaum A. Adiponectin: a manifold therapeutic target for metabolic syndrome, diabetes, and coronary disease? Cardiovasc Diabetol. 2014;13(1):103.

32. Barbieri M, Esposito A, Angellotti E, Rizzo MR, Marfella R, Paolisso G. Association of genetic variation in adaptor protein APPL1/APPL2 loci with non-alcoholic fatty liver disease. PLoS ONE. 2013;8(8):e71391.

33. Fiorentino L, Stehlik C, Oliveira V, Ariza ME, Godzik A, Reed JC. A novel PAAD-containing protein that modulates NF-kappa B induction by cytokines tumor necrosis factor-alpha and interleukin-1 beta. J Biol Chem. 2002;277(38):35333-40.

34. Lian YG, Zhao HY, Wang SJ, Xu QL, Xia XJ. NLRP4 is an essential negative regulator of fructose-induced cardiac injury in vitro and in vivo. Biomed Pharmacother. 2017:91:590-601.

35. Mehta R, Neupane A, Wang L, Goodman Z, Baranova A, Younossi ZM. Expression of NALPs in adipose and the fibrotic progression of non-alcoholic fatty liver disease in obese subjects. BMC Gastroenterol. 2014;14:208.

36. Davalos-Misslitz AC, Rieckenberg J, Willenzon S, Worbs T, Kremmer E, Bernhardt G, Förster R. Generalized multi-organ autoimmunity in CCR7deficient mice. Eur J Immunol. 2007;37(3):613-22.

37. Pei G, Yao Y, Yang Q, Wang M, Wang Y, Wu J, Wang P, Li Y, Zhu F, Yang J, et al. Lymphangiogenesis in kidney and lymph node mediates renal inflammation and fibrosis. SciAdv. 2019;5(6):eaaw5075.

38. Szanya V, Ermann J, Taylor C, Holness C, Fathman CG. The subpopulation of CD4+CD25+ splenocytes that delays adoptive transfer of diabetes expresses L-selectin and high levels of CCR7. J Immunol (Baltimore, MD: 1950). 2002;169(5):2461-5.

39. Hellmann J, Sansbury BE, Holden CR, Tang Y, Wong B, Wysoczynski M, Rodriguez J, Bhatnagar A, Hill BG, Spite M. CCR7 maintains nonresolving lymph node and adipose inflammation in obesity. Diabetes. 2016:65(8):2268-81.

40. Kim HM, Lee BR, Lee ES, Kwon MH, Huh JH, Kwon BE, Park EK, Chang SY, Kweon MN, Kim PH, et al. iNKT cells prevent obesity-induced hepatic steatosis in mice in a C-C chemokine receptor 7-dependent manner. Int J Obes. 2018:42(2):270-9.

41. Fuchs BC, Hoshida Y, Fujii T, Wei L, Yamada S, Lauwers GY, McGinn CM, DePeralta DK, Chen X, Kuroda T, et al. Epidermal growth factor receptor inhibition attenuates liver fibrosis and development of hepatocellular carcinoma. Hepatology (Baltimore, MD). 2014;59(4):1577-90.

42. Liang D, Chen H, Zhao L, Zhang W, Hu J, Liu Z, Zhong P, Wang W, Wang J, Liang G. Inhibition of EGFR attenuates fibrosis and stellate cell activation in diet-induced model of nonalcoholic fatty liver disease. Biochim Biophys Acta Mol Basis Dis. 2018;1864(1):133-42.

43. Kalucka J, BierhansI L, Conchinha NV, Missiaen R, Elia I, Brüning U, Scheinok S, Treps L, Cantelmo AR, Dubois C, et al. Quiescent endothelial cells upregulate fatty acid $\beta$-oxidation for vasculoprotection via redox homeostasis. Cell Metab. 2018;28(6):881-894813.

44. Rahman K, Liu Y, Kumar P, Smith T, Thorn NE, Farris AB, Anania FA. C/EBP homologous protein modulates liraglutide-mediated attenuation of nonalcoholic steatohepatitis. Lab Investig. 2016;96(8):895-908.

\section{Publisher's Note}

Springer Nature remains neutral with regard to jurisdictional claims in published maps and institutional affiliations. 